\title{
A Ten Year Retrospective Audit of the Clinic-pathological Management and Treatment Outcomes of Patient's with Nephroblastoma (Wilms Tumor) at Kenyatta National Hospital
}

\author{
P.M Mwamba*, E. Rogena, G.W Kitonyi \\ Department of Pathology, University of Nairobi, P.O Box 19676 - 00202, Nairobi, Kenya \\ *Corresponding author: pmmwamba@yahoo.com
}

\begin{abstract}
Background and aims: Treatment of Nephroblastoma (Wilms' tumor) in children is one of medicine's success stories. Due to improvements in surgical techniques, drug therapies, and radiation, 85 to 90 percent of children with Wilms tumor who receive state-of-the-art treatment are cured. While this is true for developed countries the outcomes of nephroblastoma (Wilms' tumor, WT) in our hospital and other developing countries are notably below those in these countries. There are continuous reviews and updates of clinical pathologic features, treatment designs, and treatment outcomes every 2 or 3 years, in the developed countries. However, in resource poor countries, such frequent reviews and updates are presently not affordable but, nevertheless, efforts should be made to undertake them every 5 or so years, to improve patient management and treatment outcomes. The objectives of this study were to review the clinical presentation and management of children with Wilms tumor and the factors influencing the outcome at Kenyatta National Referral and Teaching Hospital (KNH). This was a retrospective descriptive cross-sectional study. Analysis of the data was carried out using the statistical package for the social sciences (SPSS) software. The findings of the study will form a basis for revision of treatment design for patients diagnosed with Nephroblastoma at KNH, and by extension, other tertiary public health care facilities in Kenya. Patients and methods: The records of 140 WT patients, aged less than 16 years, who were treated in Kenyatta National Hospital, Kenya, during the period from January 1997 to December 2008 were reviewed. The management protocol followed the scheme of the US National Wilms Tumor Study Group (NSWTG). Results: Thirteen cases (38.2\%) were diagnosed as stage I, 4 (11.8\%) as stage II, $13(38.2 \%)$ as stage III and 2 (5.9\%) as stage IV. Eight cases had bilateral disease (stage V). Four-year overall survival (OS) and event free survival (EFS) rates were $\mathrm{xx} \%$ and $\mathrm{xx} \%$, respectively. Univariate analysis by Log-rank test revealed statistically significant associations between OS and nodal status ( $\mathrm{p}$ - value $<0.01$ ), manifestation of gross hematuria ( $\mathrm{p}$-value 0.02 ), and tumor size of 10 centimeters or more (p-value 0.02). Multivariate analysis found only the nodal status to be independently associated with OS at a Hazard Ratio of 16.6 (p-value < 0.01). Eight of 13 stage I cases and 6/13 stage III cases had relapsed, with two-year post-relapse survival of $42.8 \%$. Significantly poorer outcome was found in cases with early relapse within 200 days after enrollment (p-value 0.02). Conclusion: Childhood Wilms' tumor presents late in our setting with its consequent management challenges. The need to educate the populace and the primary healthcare providers on the benefits of early diagnosis and treatment of this condition cannot be overemphasized. Large tumor size and gross hematuria were associated with risk of a poorer outcome.
\end{abstract}

Keywords: nephroblastoma, Wilms' tumor, hematuria, survival, prognosis

Cite This Article: P.M Mwamba, E. Rogena, and G.W Kitonyi, "A Ten Year Retrospective Audit of the Clinic-pathological Management and Treatment Outcomes of Patient's with Nephroblastoma (Wilms Tumor) at Kenyatta National Hospital." American Journal of Cancer Prevention, vol. 5, no. 2 (2017): 21-24. doi: 10.12691/ajcp-5-2-1.

\section{Introduction}

Nephroblastoma is a malignant tumor of the kidney [1]. It is mainly a childhood cancer, even though it occurs in adults occasionally. In Kenya, Nephroblastoma accounts for $3.3 \%$ of childhood cancers [2,3,4,5]. This is a lower incidence than in Western countries where it accounts for up to $8 \%$ of childhood cancers [6]. Nevertheless, it forms a significant burden in the management of childhood cancers in Kenya. In the industrialized countries, treatment of Nephroblastoma is currently based on clinical and pathological features at presentation [8,9]. These features include the age of the patient, histologic grading and the phenotype of the tumor, anatomic extent of the disease, (i.e. stage), cytogenic and molecular characteristics of the tumor. Based on these features, patients are categorized 
into low risk, intermediate risk, and high risk groups. Low risk patients receive the least intense therapy, while high risk patients receive the most intense therapy [10,11]. Treatment includes surgery, chemotherapy, radiation therapy, singly or in combination. Using these treatment modalities, and with frequent treatment reviews and updates, survival rates of for group I patients with localized disease, a survival rate of $97 \%$ has been achieved. Significant clinical data have justified the elimination of postoperative irradiation and its deleterious long-term side effects in group I children under 2 years of age, and current studies replacing radiotherapy with combination drug therapy will probably allow the elimination of radiotherapy for all group I patients. The combination of dactinomycin-vincristine (AMD-VCR) has proven superior to single-agent treatment, and in group II and III patients with local tumor extension, survival rates have reached $86 \%$. Most remarkable is the achievement of an $83 \%$ survival rate at 2 years in group IV patients with metastatic disease at the time of diagnosis, since survival of this type of patient before chemotherapy was nil. The newly recognized histologic grading of "unfavorable" and "favorable" prognostic types of tumor should stimulate refinement of therapy and further improvement in survival by application of more or less aggressive treatment, as appropriate. The current survival rate of $87 \%$ in patients with bilateral tumors is also remarkable and underscores the need for flexibility and an understanding of tumor biology for successful clinical management.

In Kenya, Professor Kasili formulated the first treatment protocol for Nephroblastoma in the mid-seventies. Based on this protocol, all Nephroblastoma patients had a very poor prognosis, with all patients being dead within two years of diagnosis, irrespective of their age or other clinical features. Ad hoc changes to this protocol were made in the early eighties and formal revisions made in the year 2002 by Professor Macharia and Dr Mwanda [13]. No studies have been done on survival of Nephroblastoma patients since 1987, in spite of these treatment protocol changes and significant improvement in diagnostic facilities, which include introduction of immunophenotyping, imaging, (CT scans, MRI), in the country, even though these improvements are still limited. Given that it is now over 20 years since pathological and survival rates of patients with Nephroblastoma were carried out in Kenya, [3,12] and 12 years since some clinical features of patients with the diagnosis of Nephroblastoma at KNH were described [8], and in view of revised protocols and refinement in diagnostic facilities over that period, a study of clinical pathologic, treatment and treatment outcomes of patients with Nephroblastoma was clearly overdue. The results of this study could assist in designing new, or modifying the current treatment protocols, depending on the findings of the study,

\section{Patients and Methods}

This was a retrospective descriptive cross-sectional study of children aged 0-15 years admitted at the Kenyatta National Hospital with a diagnosis of histologically confirmed Wilms' tumour from January 1997 and December 2006. Kenyatta National Hospital is a national teaching and referral hospital and the main hjosptal where children with cancer are treated.

The study undertaken at the (KNH) used information recorded in patient's records between Jan 1997 and December 2006. Using the KNH record office patient data base, inpatient numbers, (IP No's), of patients admitted at KNH with diagnosis of Nephroblastoma was obtained from filed cards for the years between January 1997 and December 1999 by discharge diagnosis, (this was the period before records computerization). IP No's for patients diagnosed with Nephroblastoma for the years 2000, 2003, 2004, 2005 and 2006 were obtained from the computer records in the $\mathrm{KNH}$ records office by discharge diagnosis. With the IP No's., patients files were pulled from the shelves and scrutinized to exclude any that may have been misclassified. Using coded predesigned data extraction forms, pre-trained research assistants entered the relevant information. The information was edited by a data manager and cross-checked for accuracy. The investigator and a collaborator crosschecked every $5^{\text {th }}$ file for accuracy independently.

\section{Results}

Out of 803 children with cancer, 71 (8.8\%) had histologically proven nephroblastoma. At presentation, $1.5 \%$ were in stage I, $13.2 \%$ stage II, $36.8 \%$ stage III, 41.2\% stage IV and $7.4 \%$ stage V. Eighty five per cent presented with stage III-V disease. Ninety five per cent had nepherectomy and received chemotherapy. Radiotherapy was given to $50.7 \%$ of the patients. Nine patients died before commencement of chemotherapy, two of whom died in the immediate post-operative period. The median duration between admission and surgery was 41 days. Pre-operative chemotherapy was given to $42 \%$ of the patients. Approximately $25.5 \%$ of the patients received little or no induction chemotherapy due to unavailability of drugs while only $2.8 \%$ received the prescribed maintenance treatment with the remainder getting erratic or no treatment. Overall, only $34.7 \%$ remained disease free two years from time of diagnosis.

There were 32 children (M:F=1.9:1) with histologically confirmed nephroblastoma seen over the 7-year period. Their median(range) age was 4 (3-15) years. The patients invariably presented with a palpable abdominal mass, but haematuria was exceptional. At presentation, 1 (3.1\%) patient was in stage I, 8 (25\%) stage II, 11 (34.4\%) stage III and 12 (37.5\%) stage IV. About $72 \%$ of the patients presented with stage III-IV disease. Poorly differentiated neoplasm was more common in male than in female patients. Nephrectomy and chemotherapy were the modality of treatment. Fifteen (46.9\%) of the patients received little or no induction chemotherapy due to unavailability of drugs while only 12 (37.5\%) received the prescribed maintenance treatment with the remainder getting erratic or no treatment. Overall, only $43.8 \%$ were alive between 1 and 9 months (median: 6 months) of follow-up period, but there was no survivor at two years after treatment. 


\section{Conclusion}

Childhood nephroblastoma has a high mortality rate in north central Nigeria because of late clinical presentation with advanced disease, poor availability of cytotoxic drugs and frequent interruptions in treatment and inadequate follow-up. Late presentation, poor availability of cytotoxic drugs and frequent treatment interruptions for various reasons have contributed to the poor outcome of nephroblastoma in Kenya.

\subsection{Discussion}

Childhood Wilms' tumour is characterized with late presentation in our setting. Whereas presentation is early in the developed world, presentation is still late in the Sub-Saharan sub-region [3]. Ekenze et al reported 40 cases of late presentation in a period of 10 years [3]. Hadley et al [4] noted a similar feature of late presentation that was associated with malnutrition. These compare with our 6 years' experience with late presentation of Wilms' tumour in 34 children (only 1 child had a stage I tumour). Incidentally this period coincides with the era when medical treatment in children (aged 0-12years) was made free by the state government. Indeed it is still free for that age group.

Apart from ignorance, negligence on the part of parents may probably be one of the reasons responsible for the late presentation despite the free medical treatment for this age group in our setting. The consequences of late presentation include very huge and advanced tumours which make operative intervention very difficult and prognosis correspondingly poor. Other factors that might influence the prognosis include; inadequate drug supply (chemotherapeutic drugs are not free in our setting despite the free medical services for this age group) and comorbidities affecting the patient: malnutrition, human immunodeficiency virus/acquired immune deficiency syndrome (HIV/AIDS), tuberculosis [4]. Although these diseases may affect the prognosis, we did not routinely screen our patients for them. Operating on these patients could be challenging and daunting for the inexperienced even for the experienced surgeon. Firstly, torrential bleeding may be a problem and because the tumours are usually of very large size, considerable amount of blood may be may be lost when the tumour is resected. To minimize this blood loss intra-operatively, the renal artery should be ligated first before the renal vein (which should be ligated later after some minutes interval). This reduces the amount of stroke volume going into the mass [5]. Besides, ligating the vein later does not worsen the prognosis [6]. Secondly, because these tumours are usually large, the usual renal pelvis anatomy is distorted, the renal vessels could be obscured and displaced by the large mass, consequently, gaining vascular control becomes difficult [7]. We overcame this difficulty for the definitive operable cases by first ligating the ureter as low as possible. The proximal portion of the ligated ureter becomes a guide to the renal pelvis which lies immediately posterior to the renal artery. The latter is then ligated before the vein as earlier mentioned. Three patients had neoadjuvant chemotherapy and a subsequent nephroureterectomy. The chemotherapy regime consisted of vincristine weekly for 8 weeks, a course of daily Actinomycin D for 5 days and 2 doses of Adriamycin given 3 weekly. This regime was found to be effective in reducing the tumour mass $[8,9]$. Like most cytotoxic drugs, these drugs have their side effects but peculiar to Adriamycin is cardiotoxicity [10]. Two children had an adriamycin induced cardiomyopathy and the drug was discontinued. One of them died but the other survived with significant heart failure, he is currently out of heart failure.

Clearly, this study has some limitations. Firstly, it is a retrospective study with its attendant problems; 10 patients were lost to follow up. Secondly, although there was multidisciplinary collaboration involving paediatricians, paediatric surgeons, paediatric anaesthetiologists and paediatric histopathogist, a paediatric oncologist was not available. Ideally the work up of these patients should include computed tomography (CT) and magnetic resonance imaging (MRI) which characterize the tumours and show the extent of spread. These investigations were not done. We relied on the clinical findings, intravenous urographs and chest $\mathrm{x}$-rays for our diagnosis. Paediatric patients are given free medical treatment in our unit, however this does not include the supply of cytotoxic drugs and the consequence is poor compliance to the chemotherapeutic protocol. Patients did not have radiotherapy. It has been stated that comorbidities like malaria fever, tuberculosis (TB) and human immunodeficiency virus/ acquired immune deficiency syndrome (HIV/AIDS) may affect the prognosis in these patients [4]. We did not routinely test patients for these conditions. Finally, the histopathologist did not say whether the tumours were either favourable or unfavourable histology. This practice which has since been corrected, robbed us of information of prognostic value during this retrospective study.

\subsection{Conclusion}

- Patients with childhood Wilms' still present very late in our setting despite the free medical treatment for children in our locality. This poses management challenges.

- The need to educate the populace and the primary healthcare providers on the benefits of early diagnosis and treatment cannot be overemphasized.

- We also believe that the outcome will definitely improve if the cytotoxic drugs are available and affordable to the patients.

- In addition, the cured patient should be followed up for secondary malignancies.

\section{Acknowledgements}

The authors wish to thank the Kenyatta National Hospital records department for providing the records and files.

\section{References}

[1] Ekenze SO., Heij HA., Youngson GG.. Kidney tumours in Emmanuel Ameh et al (eds): Paediatric Surgery: A comprehensive Text for Africa. Global-HELP Organization, Seattle, WA, 2011. 
[2] Mitchell C., Jones PM., Kelsey A. et al.. The treatment of Wilms' tumour: Results of the United Kingdom Children's Cancer Study .Br J. Cancer, 2000; 83, p 602-608.

[3] Ekenze SO., Agugua-ObianyoNEN, Odetunde OA. The challenge of nephroblastoma in a developing country. Ann Oncol 2006; 17: p 1598-1600.

[4] Hadley GP, Naude F. Malignant solid tumour, HIV infection and tuberculosis in children: an unholy triad. Pediatric Surgery International 2009; 25: 697-701.

[5] Sembulingam K., Sembulingam M.. Cardiac output, in Essentials of Medical physiology, ed 5. New Delhi; Jaypee Brothers Medical Publishers (p) ltd, 2010, p 553.

[6] Leape LL., Breslow NE.. The surgical treatment of Wilms' tumour: Results of the National Wilms' Tumor Study. Ann Surg 1978; 187: p 351-356.

[7] Tagg EP., Thomas PB,Othersen HB.. Wilms' tumor in Grosfeld JL et al.(eds): Pediatric Surgery, ed 6. Philadelphia, Mosby Elsevier Yearbook Publishers, 2006, p 445-460.

[8] Isreals T., Molyneux EM., Caron $\mathrm{HN}$ et al. Preoperative chemotherapy for patients with Wilms' tumor in Malawi is feasible and efficacious. Pediatric Blood and Cancer 2009, 53(4); 584-589.

[9] Tournade MF., Com-Nougue C., de Kraker J et a.. Optimal duration of pre-operative therapy in unilateral and non-metastatic Wilms' tumour in children older than 6 months: results of the ninth International Society of Pediatric Oncology Wilms' tumour trial and study. J.Clin Oncology 2001; 19, p 488-500.

[10] Goorin AM., Borrow KM., Goldman A et al. Congestive heart failure due to Adriamycin cardiotoxicity: Its natural history in children. Cancer 47:2810, 1981.

[11] Macharia WM'; Childhood cancers in a referral hospital in Kenya: a review. Journal: East Afr Med J. 1996 Oct;73(10):647-50.

[12] Abdallah FK, Macharia WM Clinical presentation and treatment outcome in children with nephroblastoma in Kenya. East Afr Med J. 2001 Jul;78(7 Suppl): S43-7.

[13] Kitonyi GW1, Macharia WM, Mwanda OW, Pamnani R.; Clinicopathologic characteristics and treatment outcomes in children with neuroblastoma at the Kenyatta National Hospital, Nairobi. East Afr Med J. 2009 Dec;86(12 Suppl): S39-46. 